\title{
Aprendizaje Servicio Universitario: creando empleo a partir de la emprendeduría social
}

\author{
Salvador Simó Algado \\ Universidad de Vic (España) \\ salvador.simo@uvic.cat \\ Xavier Ginesta Portet \\ Universidad de Vic (España) \\ xavier.ginesta@uvic.cat \\ Jordi De SAN Eugenio Vela \\ Universidad de Vic (España) \\ jordi.saneugenio@uvic.cat
}

\section{Resumen}

La Universidad debe erigirse como motor de la sociedad civil y abordar con decisión los desafíos sociales contemporáneos, como es la situación de paro en España. La metodología del Aprendizaje Servicio Universitario permite integrar un aprendizaje basado en la realidad con el servicio a la comunidad concretizando la responsabilidad social de la Universidad. El artículo presenta el proyecto interfacultativo EcoSPORTech desarrollado en la Universidad de Vic cuya finalidad ha sido la creación de empleo juvenil desde la emprendeduría social. La política de comunicación ha sido clave en este proyecto.

Palabras clave: Emprendeduría, aprendizaje servicio, desempleo juvenil, responsabilidad social universitaria, comunicación.

\section{University Service Learning methodology: creating employment with social enterpreneurship}

\begin{abstract}
The University must become an engine of the civil society and take an active part in leading contemporary social challenges, such as the situation of unemployment in Spain. The University Service Learning methodology allows us to integrate a reality-based learning with the community service, concretizing the social responsibility of the University. The article presents the interfacultative EcoSPORTech project developed at the University of Vic. The purpose of this project has been the creation of youth employment using social entrepreneurship. The communication policy has been a key factor in this project.
\end{abstract}

Key Words: Entrepreneurship, service learning, young unemployment, social responsibility, communication.

\section{Referencia normalizada:}

Simó Algado, S.; Ginesta Portet, X. y San Eugenio Vela, J. (2013) Aprendizaje Servicio Universitario: creando empleo a partir de la emprendeduría social. Historia y Comunicación Social. Vol. 18. No Especial Noviembre. Págs. 627-638. 
Sumario:_1.Introducción y estado de la cuestión. 2. Metodología. El aprendizaje servicio universitario y la emprendeduría social. 3. Desarrollo y resultados. 3.1. El proyecto EcoSPORTech. 3.1.1. La formación teórica. 3.1.2. El trabajo de campo. 3.2. La Universidad como motor de la sociedad civil, tejiendo alianzas para construir un nuevo futuro. 3.3. La comunicación al servicio de la sociedad. 4. Discusión. 5. Conclusiones. 6. Bibliografía.

\section{Introducción y estado de la cuestión}

La sociedad actual no ofrece un futuro a la mayoría de los jóvenes, como lo muestra el $50 \%$ de paro juvenil. Sin un posible trabajo los jóvenes no pueden controlar sus propias vidas, siendo pobres en el sentido apuntado por Amartia Sen (2000). Tampoco serán capaces de desarrollar un proyecto de vida significativo. Frankl (1964) ya nos advirtió que las consecuencias de la falta de sentido son la adicción, la agresión o la depresión y/o el suicidio, sin dudas grandes lacras de la sociedad actual.

Esta situación, ¿nos debe interpelar como Academia? Como dice Boff (2000) el sufrimiento más que la admiración hace pensar. No en vano, Said (2006) cuestiona el rol de los intelectuales que por interés callan ante estas realidades, cometiendo un delito de silencio. Vivimos inmersos en una modernidad líquida (Bauman, 2005), marcada por el declinar de la ciudadanía (Castells, 2005) y una grave crisis económica, ecológica y social, donde grandes sectores de la población quedan excluidos del supuesto progreso. El paro convive con una precariedad laboral, en un proceso de brasileñización del mercado laboral europeo (Beck, 2000). Un trabajo que pierde sus referentes éticos y provoca la corrosión del carácter (Sennett, 2006). Los ciudadanos afrontan estos riesgos desde la soledad, desde un atomismo social, y sin la protección de un Estado del Bienestar en proceso de desmantelamiento (Bourdieu, 1999).

Ante este escenario la Universidad debe erigirse como motor de la sociedad civil y abordar con valentía los retos globales de nuestra época como el desempleo juvenil. Debe formar profesionales capaces de trabajar en equipos multidisciplinares, desde una comprensión de la compleja problemática actual. El objetivo no se limita a comprender el mundo, sino a transformarlo (Marx, 1988).

Así, los documentos oficiales expresan la determinación de crear una Universidad comprometida con la sociedad. Harvaky (2006) analiza los objetivos fundacionales de las universidades, donde se considera la educación como la base de todo desarrollo democrático, con el servicio a la sociedad como propósito principal. Pero, la Universidad se enfrenta a una serie de obstáculos para la realización de su misión democrática: 1) Platonismo: La teoría platónica de la educación era elitista e idealista. El objetivo era la ciencia pura, en contraste con la práctica en el mundo real, considerada como inferior. 2) La comercialización: La guerra fría aceleró la comercialización de las universidades. 3) El etnocentrismo de las disciplinas: Los profesores sirven a los intereses, sin responsabilidad alguna sobre la ciudadanía, impidiendo la cooperación interdisciplinaria. 
¿Estamos preparando a los estudiantes como ciudadanos globales para el nuevo orden mundial, conceptual y éticamente? En general, el currículo no recoge temas globales, como la crisis ecológica o social, manteniendo así a las nuevas generaciones en la ignorancia (Thibeault, 2006). La educación debe desarrollar un conocimiento capaz de abordar los problemas globales para inscribir allí los conocimientos parciales y locales (Morin, 2002). Se impone la educación para una ciudadanía cosmopolita, arraigada en el local, inspirados por Kant(1946) quien soñaba con la unificación perfecta de la especie humana a través de una ciudadanía común. Es fundamental que los estudiantes sean capaces de entender el contexto social y político que determina sus vidas. Leer el mundo es un acto anterior a la lectura de la palabra. No existe en ningún momento tiempo-espacio, la existencia de una práctica educativa indiferente a propósitos sociales y políticos (Freire, 2003).

\section{Metodología. El aprendizaje servicio universitario y la emprendeduría social}

El Aprendizaje Servicio Universitario (ApS) es una metodología que nos ayuda a crear una Universidad comprometida con la sociedad. Siguiendo el trabajo de Manzano (2010) podemos afirmar que el modelo de ApS integra la teoría con la práctica, la formación con el compromiso (Speck, 2001), la cognición con la emoción (Butin, 2006). El ApS es un método de enseñanza que orienta los logros académicos hacia la construcción de ciudadanía a través del servicio comunitario (Coles, 2005). Podríamos definir el ApS como un programa de servicio solidario protagonizado por los estudiantes, orientado a atender eficazmente necesidades de una comunidad, y planificado de forma integrada con los contenidos curriculares con el objetivo de optimizar los aprendizajes (Ramson, 2009). La mayor parte de la literatura muestra los efectos en los estudiantes (eficacia personal, identidad, desarrollo moral), sociales (reducción de estereotipos, comprensión intercultural, responsabilidad social, habilidades ciudadanas, compromiso), de aprendizaje (resultados académicos, aplicación de aprendizajes en el mundo real, pensamiento crítico, análisis de problemas, comprensión de la complejidad). Las investigaciones del primer autor han manifestado como afecta positivamente no sólo a la educación y al sentimiento de ciudadanía, sino al bienestar de los estudiantes (Simó Algado, 2010).

Este aprendizaje con un alto contenido ético, en valores, tiene más sentido en el marco de una sociedad hedonista que reduce al bios politikos en un homo consumens, marcada por una ética laxa que conduce al ocaso del deber(Lipovetsky, 1994), basada en el culto al ego. Parece como si aquellos valores que hacen habitable el mundo poco a poco se van descomponiendo, bajo la acción del fermento del consumismo y del individualismo. En este contexto educar en una ciudadanía cosmopolita parece insensato. Pero, como indicó Kant (1991) hemos de educar pensando en la mejor situación posible en el futuro. No hay mejor manera de materializar un ideal que educar para conseguirlo. Tiempo para forjar el carácter de los estudiantes desde 
la ética, desde la moral. No se puede transformar el mundo desde la desmoralización, sino desde personas que pasan de espectadores a actores morales.

En este contexto, debemos mencionar igualmente la Emprendedoría Social (ES). Larsen (2012) identifica al menos 37 definiciones de ES, con denominadores comunes, tales como la creación de valor social a través de la innovación y la misión social. Siguiendo a Marques (2012), entenderemos como ES las iniciativas que se producen en cualquier sector de actividad (privada, cooperativa y social, pública, o una combinación de ambos) que presenten las siguientes características: a) con una misión que tiene como objetivo atender las necesidades sociales y está dirigido a crear, mantener y maximizar el valor social; b) que buscan promover la independencia, empoderamiento y la rendición de cuentas de los beneficiarios (personas físicas y/o grupos), buscando la transformación social; c) con un propósito y/o un proceso marcado por la innovación social; d) con una estrategia de sostenibilidad financiera, en particular mediante la diversificación y la combinación de fuentes de ingresos (los ingresos autogenerados, la filantropía/patrocinio, la financiación pública) y, finalmente, e) que evalúan y difunden los resultados e impactos sociales, financieros y de gestión de los recursos, de rendición de cuentas y de transparencia.

\section{Desarrollo y resultados}

\subsection{El Proyecto Ecosportech}

Fruto de esta reflexión, en 2012 nació un proyecto de ApS Universitario interfacultativo desde la Facultad de Empresa y Comunicación (FEC), la Facultad de Ciencias de la Salud (FCSB) y la Facultad de Educación (FE) de la Universidad de Vic (UVic), con el fin de crear una empresa social con jóvenes. La idea central del proyecto era abordar la situación de paro juvenil en España (56,5\%, en julio de 2013) desde una transferencia de conocimientos a partir de los distintos grupos de investigación de dichas facultades para poder crear un empresa dedicada a la realización de actividades de deporte y ocio en el medio natural, integrando las nuevas tecnología, de ahí su nombre EcoSPORTech. Este proyecto también busca la promoción económica de la ciudad, Vic, vía la potenciación del turismo; la participación social intergeneracional; el mejorar la salud de la ciudadanía creando espacios de deporte en el medio natural; así como la conexión de la ciudad con el bosque de ribera.

La fase inicial del proyecto implicó una sucesión de reuniones de coordinación, en el seno de la propia Universidad, y con el resto de instituciones participantes. Los participantes fueron derivados por el Área de Servicios Sociales del Ayuntamiento. Todos ellos han sido de origen inmigrante, no porque fuera un criterio de inclusión sino porque presentaban una mayor fragilidad social. Cinco de los jóvenes son de nacionalidad marroquí, uno mejicano y otro es senegalés. Han participado 6 chicos y una chica. 


\subsubsection{La formación teórica}

Una educación de calidad es clave en el proceso de inserción laboral de los jóvenes, más cuando en su mayoría presentaban graves carencias educativas. Durante un mes, los jóvenes han recibido una formación en la Unidad de Emprendedoría Social de la UVic. En la formación han participado profesores de las Facultades de Empresa y Comunicación, de Salud y de Educación. La formación se ha basado en temas relacionados con: nuevas tecnologías, medios de comunicación, emprendedoría social y creación de empresas sociales, la importancia de la ocupación para la salud, cómo evitar lesiones deportivas, nutrición y deporte, cómo crear circuitos deportivos en el medio natural, praxis en el medio natural sostenibles. Es de destacar que se ha producido una transferencia de conocimiento de diversos grupos de investigación a la formación de los jóvenes.

Se han desarrollado prácticas de comunicación en los platós de televisión del Servicio de Audiovisuales de la UVic. Además se han realizado sesiones prácticas en el medio natural, que han consistido en la creación de circuitos deportivos y de salud, pruebas de orientación y de geocaching.

\subsubsection{El trabajo de campo}

El trabajo de campo ha durado dos meses con la participación de estudiantes de terapia ocupacional y fisioterapia. Durante el mismo los jóvenes han percibido una beca de aprendizaje. Las labores han sido dirigidas por paisajistas y expertos en desarrollar actividades deportivas en el medio natural. En primer lugar se creó una conexión con el anillo verde de la ciudad de Vic, para conectar los jardines de la ciudad con el bosque de ribera. Para tal fin, se construyó un camino y se plantaron 60 tilos en el mismo. Esto ha permitido crear un circuito (de los 10.000 pasos) que va desde el Puente del Queralt, localizado en el centro de la población, al Puente del Bruguer, en el bosque de ribera. Su finalidad es fomentar el deporte y el wellness de la ciudadanía.

La siguiente intervención fue la creación del Jardín del Claustro. Se trata de un espacio de gran valor histórico y artístico, en la Catedral de Vic, en un claustro que data de los siglos XII y XIV con una parte románica y otra gótica. En este se encuentra la tumba del famoso filósofo Jaime Balmes. La intervención ha recuperado los parterres tradicionales del mismo. Se ha basado en la eliminación de malas hierbas, instalación de geo-textil en los parterres, la poda de los árboles y arbustos existentes, la plantación de un olivo, cipreses, abelias, romero, lavanda, hortensias y de planta de temporada. Es de destacar la recuperación de antiguas rejas de la catedral, que se han integrado en el diseño del jardín y que sirven de base a rosales trepadores.

Posteriormente, se creó el Jardín de las Artes, localizado frente al Centro de Artes Escénicas de la ciudad (L'Atlàntida). Este edificio es el icono del Vic moderno. Por ello se optó por una intervención sutil, que no robara protagonismo al edificio. Se plantaron cercis, lavandas, romero, rosales, así como un ginkgo biloba. En la planta- 
ción participaron más de cien niños de P3 de la escuela El Escorial, ya que una de las claves del proyecto es la participación de toda la ciudadanía.

Precisamente buscando el protagonismo de los jóvenes se ha creado el Jardín de las Identidades, basado en grafitis. Veinticinco grafiteros de la ciudad han participado en su creación. Este espacio será la base para una zona de escalada. Es de gran importancia promover la participación ciudadana de este colectivo, ante la apatía que muestra, así como crear espacios públicos que los jóvenes sientan como propios. El grafiti posee una gran importancia simbólica, ya que denota el deseo de los artistas de expresar su identidad y dejar su impronta en el mundo. Como bien decía Arendt (1997) el ser humano se inserta en el mundo a través de su palabra y de su acción. Por ello es fundamental facilitar esta inserción en el mundo de los jóvenes, que actualmente se encuentran con una sociedad cerrada.

La primera fase se ha cerrado con la creación del Jardín Filosófico, que combina el paisajismo con el arte. Se trata de la creación de un jardín de luz en el marco del Parque Balmes, dedicado al filósofo. La intervención ha consistido en la creación de la obra El corazón del Cielo, el corazón de la Tierra. Se trata de un jardín de luz formado por 12 columnas. Seis son de color azul, simbolizan el cielo, el mundo de la ideas. Seis son de color verde, y simbolizan la Tierra. Cielo y Tierra que dialogan en el jardín. Las columnas además muestran citas de Balmes.

3.2. La Universidad como motor de la sociedad civil, tejiendo alianzas para construir un nuevo futuro

El proyecto ha implicado la creación de alianzas, en primer lugar en el seno de la propia universidad, superando el etnocentrismo disciplinario. Así se estableció una colaboración entre la FEC, la FCSB y la FE de la UVic, tanto a nivel de docentes e investigadores, como de estudiantes. En segundo lugar gracias a la creación de una alianza estratégica entre la sociedad civil, el sector público y el sector empresarial. Del sector público destaca la participación del Ayuntamiento de Vic. La selección de los jóvenes participantes ha sido realizada a través de los Servicios Sociales. Asimismo no podemos obviar su participación en la financiación del mismo, con una aportación cercana a los 10.000 euros en su fase inicial.

Del sector empresarial, destaca la participación de la Obra Social de la Caixa, ya que el proyecto se inició gracias a la subvención de 23.200 euros, conseguida a través de la convocatoria competitiva de Proyectos de Inserción laboral de la citada entidad. Completa la aportación del sector empresarial la participación de la empresa Vicreu, con una donación en materiales de jardinería cercana a los 3.000 euros.

La Universitat de Vic ha sido la institución clave de la sociedad civil, como coordinadora del proyecto. Debemos mencionar la participación de la Asociación Tapís, que trabaja con jóvenes en situación de exclusión social; así como de la Fundació Centre Mèdic Psicopedagògic d'Osona (FCMPPO), institución especializada en salud mental. Es de destacar que el proyecto ha priorizado la inversión en empresas del territorio, en especial entidades de economía social. Así el trabajo de recuperación 
de metal lo ha hecho precisamente Tapís, y el trabajo de madera se ha encargado a la empresa Areté, que depende de la FCMPPO.

\subsection{La comunicación al servicio de la sociedad}

La asignatura de Taller de Producción de Comunicación -en cuarto de Periodismo - ha sido el entorno donde los estudiantes de Periodismo han trabajado con la información generada por EcoSPORTech. Esta ha podido ser: información sobre el inicio del proyecto, incorporación de nuevos partners y empresas, inicio y fin de alguna de las intervenciones en particular (por ejemplo, el Jardín del Claustro...), etc.

Esta asignatura vehicula, desde el curso 2012-13, la redacción conjunta que actualiza los medios propios de la Facultad de Empresa y Comunicación de la UVic. Estos medios de comunicación son: diario digital Sense Boira, Blog UVica't y canal de Youtube UVic. De ellos, el más interesante es el diario digital, ya que se publica en colaboración con uno de los medios en papel más importantes de la zona, el periódico regional El 9 Nou. Éste es el socio tecnológico de la Facultad a la hora de publicar digitalmente el diario de los estudiantes.

El Taller de Producción de Comunicación es una asignatura anual, que se imparte a razón de dos horas a la semana los viernes al mediodía. Los estudiantes que se matriculan se dividen en equipos de trabajo, cada uno asignado a un medio de comunicación de la Facultad, y que representan la estructura de una redacción. Estos grupos se van intercambiando los medios donde publicar, cada quince días uno de diferente.

La valoración que se hace de este primer año de funcionamiento es muy buena. De hecho, EcoSPORTech permite a los estudiantes de Periodismo trabajar información generada por un proyecto transversal de ciudad, donde participan actores académicos, políticos y agentes económicos del territorio. La información, pues, tiene diversos matices y, por lo tanto, contribuye a una formación transversal de las habilidades periodísticas de los estudiantes. Además, el hecho de trabajar con tres medios ya nacidos en el entorno 2.0, permite a los estudiantes desarrollar aquellas habilidades vinculadas al ciberperiodismo y a los entornos transmedia.

A parte de las informaciones publicadas por los estudiantes, los profesores también han participado de la promoción de este proyecto con artículos de fondo en algunos de los periódicos de referencia en la zona (El 9 Nou) y en Cataluña (El Punt Avui).

\section{Discusión}

El proyecto continúa con la creación de nuevos jardines y espacios deportivos en la ciudad, en el marco del proyecto Vic: paisajes con alma. Se espera durante 2013 crear dos nuevos jardines. Mientras, se ultiman los planes de viabilidad y de empresa para ver si es posible crear la empresa social EcoSPORTech. Esta sería sin duda la finalidad última del proyecto, desde la emprendedoría social. Esta empresa pasaría a 
formar parte del vivero de empresas sociales de Creacció, la Unidad de Emprendedoría de la UVic y el Ayuntamiento de Vic.

De todos modos, con lo hecho hasta día de hoy, podemos apuntar a algunas ideas interesantes para la reflexión. El paso de una universidad platónica, encerrada en sí misma, a una universidad al servicio de la sociedad, implica un proceso de transformación. Si bien los discursos y los documentos universitarios van en esta dirección, las estructuras no están muchas veces preparadas para tal fin.

El trabajar desde alianzas con diversos interlocutores añade dificultad al aumentar el número de instituciones participantes, cuyas finalidades y objetivos no siempre coinciden. Por ello es fundamental ser conscientes de que ningún objetivo importante se puede conseguir desde la soledad, y desarrollar un buen liderazgo capaz de identificar las fortalezas que cada contraparte puede aportar al proyecto, limar las diferencias de interés y establecer un objetivo compartido.

Uno de los aspectos más complicados del proyecto ha sido su financiación. Por ello es fundamental apuntar a fórmulas mixtas entre capitales públicos y privados, así como a la generación de ingresos propios.

El trabajo multidisciplinar es todavía una asignatura pendiente, más cuando hablamos de un trabajo interfacultativo. El proyecto ha aportado una sinergia entre la FEC, la FCSB y la FE, inédita hasta el momento en la UVic. Ha mostrado el gran potencial que se despliega merced a este tipo de colaboraciones. Ciertamente la innovación se encuentra muchas veces entre las fronteras de los distintos saberes. Por ello se impone la necesidad de abandonar caminos más seguros y conocidos, para aventurarnos en la exploración de las nuevas y fascinantes posibilidades que nos ofrece la multidisciplinariedad.

Las edutrópolis son un aspecto clave de la creación de marca de los distintos territorios. Pero esta marca no puede dejar de lado los aspectos de bienestar de sus habitantes. En este sentido, el proyecto EcoSPORTech tendrá un papel importante en el proyecto del Ayuntamiento de Vic Conceptualización y creación de la 'Marca Vic', proyecto que se desarrollará durante el curso 2013-2014 y tiene la UVic como socio estratégico: tiene por objetivo el diseño y creación de una marca para la ciudad de Vic, partiendo del análisis de su base social para así crear una marca integradora que permita la promoción de la ciudad más allá del turismo (San Eugenio, 2012).

Para los estudiantes, EcoSPORTech supone una gran riqueza, no sólo por el contacto con otros saberes, sino sobretodo por desarrollar un aprendizaje basado en la realidad. Los conceptos abstractos se encarnan en el campo de la praxis. La reflexión y la acción fluyen con naturalidad

En especial es de gran importancia empoderar la relación entre los ámbitos de empresa y comunicación, con los ámbitos sociales y de salud. Cada vez más se precisan formulas de emprendimiento social para poder financiar proyectos sociales y de salud ante la crisis y desmantelamiento del Estado Bienestar. La emprendedoría social aparece como una fórmula importante para hacer frente a dicha crisis. Pero es 
importante afirmar que la emprendedoría social no puede sustituir al Estado en su responsabilidad social.

Es fundamental el visibilizar las problemáticas sociales y de salud contemporáneas, no en vano el ser humano es un ser capaz de palabra, acción y narración (Ricoeur, 2005). El proyecto ha supuesto para los estudiantes de comunicación el contacto directo con realidades desconocidas para muchos de ellos, más allá de los propios medios de comunicación. Les ha permitido reflexionar sobre cómo comunicar las mismas sin caer en el sensacionalismo ni el victimismo de dichos colectivos, así como en la responsabilidad social y ciudadana de los medios de comunicación. Dichos proyectos sociales y de salud requieren de una buena política de comunicación desarrollada por profesionales que tengan un conocimiento real de las mismas.

La creación de trabajo, en especial para los jóvenes, es uno de los retos más importantes que tenemos como sociedad. Vivimos en una sociedad que se cierra a los jóvenes. Las consecuencias se manifiestan en el aumento de la exclusión social, los problemas de salud y la desafección ciudadana, germen de cultivo de fascismos y movimientos radicales antidemocráticos como tristemente ha demostrado la historia.

\section{Conclusiones}

Como nos enseñó Bohm (2001), el problema es que somos repetitivos cuando debemos ser creativos. En el contexto de crisis actual no nos podemos permitir desaprovechar el ingente capital social e intelectual que reside en las universidades.

La alianza entre la sociedad civil, de la que la universidad debe ser un motor destacado, junto al sector empresarial y al sector público, se manifiesta como una gran oportunidad para abordar la grave crisis social y económica que enfrentamos.

La universidad puede y debe abandonar su torre de marfil para generar un conocimiento transformador desde la propia realidad, dando un servicio a la sociedad, en especial a los colectivos más frágiles.

La estrategia de ApS Universitario puede ser un gran recurso para tal fin, donde los estudiantes integran conocimientos al tiempo que dan un servicio a la sociedad desde una mirada multidisciplinar. En este sentido, el trabajo multidisciplinar se ha visto reflejado con la participación de estudiantes de diversas facultades en el proyecto, unos trabajando como terapeutas y los otros como comunicadores de una realidad que, seguramente, hubiera pasado desapercibida por los medios de comunicación tradicionales. Aquí, tanto el uso de medios propios de la UVic, vinculados con el entorno 2.0, así como la sinergia establecida entre la FEC y la empresa El 9 Nou socio tecnológico para la revista digital de la facultad-han sido claves para dar salida a las informaciones semanales generadas por EcoSPORTech. 
Es básico el crear empresas cuya principal misión sea maximizar el beneficio social. Por ello, la emprendeduría social se erige como una estrategia clave ante los desafíos sociales y ecológicos que confrontamos. El proyecto tiene el valor fundamental de crear espacios donde los jóvenes pueden desarrollar sus potencialidades y conectar con la sociedad. Si bien el grupo de jóvenes ha mostrado ciertas carencias propias de la falta de una buena formación previa, han mostrado una gran capacidad y sus ganas de participar en la comunidad. Sin duda la sesión más especial para ellos fue la sesión donde crearon el Jardín de las Artes conjuntamente con los niños del Colegio Escorial. Han manifestado sus ganas de insertarse en el mundo laboral así como las capacidades que tienen para hacerlo, tan sólo les faltan oportunidades.

Este impacto también es positivo para nuestros universitarios, que no escapan a la preocupación por la situación actual del país, al sentimiento de desesperanza y nihilismo que se ha instalado en la sociedad, donde parece que emigrar va a ser la única opción viable para la mayoría si quieren un trabajo digno. El proyecto les permite realizar una acción inteligente (Dewey, 1969), el comprender una realidad compleja, desde una mirada multidisciplinar; para luego desarrollar acciones transformadoras, en la creación de una comunidad inclusiva y sostenible. Es un espacio para la reflexión sobre principios como la justicia social, los derechos humanos o la ciudadanía. Es fundamental el aprendizaje sobre su propia capacidad de mejorar la situación actual. Hay que alimentar la esperanza._Por ello, es vital desarrollar proyectos de inserción laboral para jóvenes, para que puedan desarrollar un proyecto de vida significativo y participar como lo que son, ciudadanos de pleno derecho. En febrero de 2013 el proyecto ha sido reconocido como Good practice por la UNESCO a través de la Global University Network for Innovation.

\section{Bibliografía}

ARENDT, H. (1997). ¿Qué es la política? Barcelona: Paidós.

BARBER, B. (2000). Un lugar para todos. Barcelona: Paidós.

BAUMAN, Z. (2005). Vidas desperdiciadas. La modernidad y sus parias. Barcelona: Paidós.

BECK, U. (2000). Un nuevo mundo feliz. Barcelona: Paidós.

BOFF, L. (2000). La dignidad de la tierra. Madrid: Trotta.

BUTIN, D. (2006). "The limits of service-learning in higher education". En: The Review of Higher Education, Vol. 29, Baltimore: The Johns Hopkins University Press. p. 473-498.

CAMUS, A. (2002). El hombre rebelde. Barcelona: Alianza.

CASTELLS, M. (2005). "Global governance and global politics". En: Political Science and Politics, Vol. 38, nº 1 , Cambridge: Cambridge Journals. p 9-15.

COLES, E. (2005): A Why do service-learning? Issues for first-time faculty. En: BELLNER, M. \& POMERY, J. (eds.) (2005). Service-Learning: Intercommunity 
\& Interdisciplinary Explorations. Indianopolis: University of Indianopolis Press. p. 85-95.

DEWEY, J. (1969). The ethics of democracy. Carbondale: Southern Illinois University Press.

El País, 30-11-06.

FRANKL, V. (1964). El hombre en búsqueda de sentido. Barcelona: Herder.

FREIRE, P. (2003). El grito manso. Buenos Aires: Siglo XXI.

HARKAVY, I. (2006): "The role of the universities in advancing citizenship and social justice in the 21st century". En: Education, citizenship and social justice, Vol. 1, $\mathrm{n}^{\circ}$ 1, Londres: Sage. Disponible en: http://esj.sagepub.com. [05-01-2007].

KANT, I. (1946). Lo bello y lo sublime. Madrid: Espasa Calpe.

KANT, I. (1991). Sobre pedagogía. Vic: Eumo Editorial.

LARSEN, M. (2012). "Social Entrepreneurship-A single case study of a social entrepreneur in the Danish onot-for-profit sector". Aahrus. Aahrus University. Department of Business Administration. Master thesis. Disponible en

http://pure.au.dk/portalasbstudent/files/44653343/Social_Entrepreneurship_A_ single_case_study_of_a_social_entrepreneur_in_the_Danish_not_for_profit_sector.pdf [0-용-2012].

LIPOVETSKY, G. (1994). El crepúsculo del deber. Barcelona: Anagrama.

MANZANO, V. (2010). "El APS y su potencial para la educación superior". En VI Jornadas de educación en Psicología. Universidad de Sevilla, 15 de junio de 2010.

MARX, K. (1988). Textos cardinales (Edición de Jacobo Muñoz). Barcelona: Ediciones Península.

MARQUES, M. (2012). "Emprendedorismo social: do enquadramento teórico à realidade portuguesa". Covilha. Universidade da Beira Interior. Master thesis. Disponible en http://personales.ya.com/aeca/pub/on_line/comunicaciones_xivencuentroaeca/cd/122h.pdf [03-07-2012].

MORIN, E. (2002). Los siete saberes necesarios para la educación del futuro. Barcelona: Paidós.

NGAI, S. (2006). "Service-learning, personal development, and social commitment". En: Journal of Adolescence, Vol. 41, nº161, Londres: Elsevier. p. 165-176.

RANSOM, L. (2009). "Sowing the seeds of citizenship and social justice". En: Education, citizenship and social justice, Vol. 4, Londres: Sage. p. 211-224.

RICOEUR, P. (2005). Caminos del reconocimiento. Madrid: Trotta.

SEN, A. (2000). Desarrollo y libertad. Barcelona: Planeta.

SENNETT, R. (2006). La corrosión del carácter. Barcelona: Anagrama.

SAID, E. (2006). Representaciones del intelectual. Barcelona: Paidós.

SAN EUGENIO, J. de (2012). Teoria i mètodes per a marques de territorio. Barcelona: UOC.

SIMÓ, S. (2011). La palabra y la acción. Vic: Universitat de Vic. Tesis doctorals en xarxa. Disponible en http://tdx.cat/bitstream/handle/10803/9325/PALABRAACCION.pdf?sequence $=1$ [20-10-2013].

SOUSA, B. (2005). El milenio huérfano. Madrid: Trotta. 
SPECK, B. (2001). "Why Service-Learning?" En: New Directions for Higher Education, Vol. 114, San Francisco: Wiley. p. 3-13.

THIBEAULT, R. (2006). "Globalization, universities and the future of occupational therapy: Dispatches from the majority world". En: Australian Occupational Therapy Journal, Vol. 53, Sydney: Wiley. p. 159-165.

\section{Los autores}

Salvador Simó Algado (Barcelona, 1973) es doctor en Educación Inclusiva por la Universidad de Vic, y profesor agregado e investigador en dicha universidad. Es máster en Dirección de Empresas y licenciado en Terapia Ocupacional. Actualmente es consultor internacional y profesor de máster en las universidades de Porto, La Sorbona-París, y Oxford Brookes; así como Director del Postgrado internacional de Emprendeduría Social. Es autor de diversos libros y artículos en revistas de ámbito internacional.

Xavier Ginesta Portet (Balenyà, 1983) es doctor en Comunicación y Periodismo por la Universidad Autónoma de Barcelona y profesor agregado en la Universidad de Vic. Es periodista y analista político en El Punt Avui y la versión digital de El Periódico de Catalunya. También, colabora con El 9 Esportiu y El 9 Nou. A nivel académico ha publicado en revistas referentes del sector de la comunicación, tanto en América como en Europa. Ha sido profesor invitado en la Universidad de Kalmar, Bonn, O Minho y Católica de Chile.

Jordi de San Eugenio Vela (Girona, 1975) es doctor en Comunicación Social por la Universidad Pompeu Fabra. Profesor del Departamento de Comunicación de la Universidad de Vic. Licenciado en Geografía y Periodismo, es también máster en Medio Ambiente. Sus intereses de investigación son cercanos a los procesos de comunicación que se llevan a cabo desde el territorio, con especial incidencia en el turismo, el paisaje y las marcas de lugar. Con relación a estos temas, ha publicado libros, capítulos de libros y artículos en revistas de ámbito internacional 